\title{
Biometric Human Recognition using ECG Signals
}

\author{
Nisha Yadav ${ }^{1}$, Manoj Duhan ${ }^{2}$, Anil Rose ${ }^{3}$ \\ M.Tech Scholar, Electronics \& Communication Engineering Department, Deenbandhu Chhotu Ram University of \\ Science and Technology, Murthal, Sonepat ${ }^{1}$
}

Professor, Electronics \& Communication Engineering Department, Deenbandhu Chhotu Ram University of Science and Technology, Murthal, Sonepat ${ }^{2}$

Associate Professor, Electronics \& Communication Engineering Department, Chandigarh College of Engineering and Technology, Chandigarh ${ }^{3}$

\begin{abstract}
In this research work, ECG of 10 subjects is recorded using the BIOPAC MP 100 system with Acknowledge software. The recorded signal is pre-processed to remove the unwanted high frequency signal, so it is filtered using Low Pass FIR filter with $40 \mathrm{~Hz}$ cutoff frequency. Statistical Parameters such as mean, variance, standard deviation etc are used to support the uniqueness property of an ECG signal for each subject. The classifier used in this research work is the Artificial Neural Network (ANN), which is trained with the extracted features. The error in prediction of the ECG of 10 subjects is nearly zero. So the accuracy of the identification system is $100 \%$.
\end{abstract}

Keywords: Biometric Characteristics, Biometric Traits, Electrocardiogram (ECG), Artificial Neural Network (ANN), Human Recognition.

\section{INTRODUCTION}

The heart is a powerful muscle that pumps blood throughout the body by means of a coordinated contraction [6]. The heart is able to conduct the signals. A natural electrical system causes the heart muscle to contract. Electrical currents that are generated by the heart as it beats spread not only within the heart, but also throughout the body. The ECG signal is the electrical variation caused by the contraction and relaxation of the heart muscle [8]. ECG waveforms depends human heart and body anatomy.

Identification of any human is generally based on its behavior and physiological traits. Physiological traits may be finger print, hand geometry, Iris pattern, EEG \& ECG etc. The ECG signal may contain significant pointers to distinguish and identify persons. Heart beat is the symbol of life and ECG is the measure of the electrical activities of the heart. It does not depend on the external body traits, it's an internal trait therefore it is more secure than any other traits. ECG signal is mainly used to find out the heart related diseases but from past decade there are many research papers that demonstrate the use of electrocardiogram as a biometric trait. There is a basic waveform of an ECG signal that can be present in every human. Figure 1 shows the basic structure of an ECG waveform.

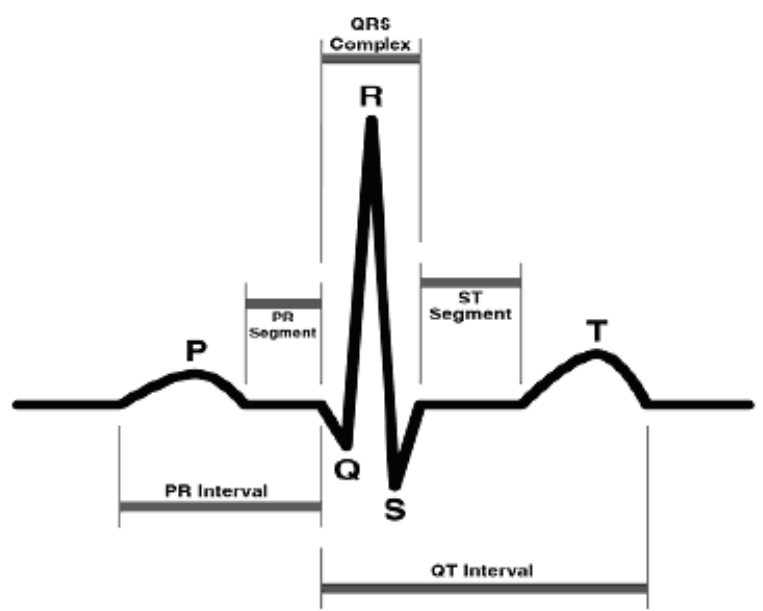

Figure 1: Basic ECG waveform [4] 
It consists of $\mathrm{P}, \mathrm{QRS} \& \mathrm{~T}$ waves. ECG signal provides the information about the electrical activities of the heart by altering the shape of these waves [2]. In ECG, the shape of the waves can also be changed by some heart disease and abnormalities [4].

\section{BIOMETRIC CHARACTERISICS OF ECG}

Biometric is defined as the automatic identification or identity verification of living persons using their enduring physical or behavioral characteristics [1] [8]. The characteristics of any biometric identification system are:-

1. Universal: Each person should possess the biometric trait.

2. Unique: Biometric trait should be unique for each person i.e. no two individuals have the same characteristics.

3. Permanent: Biometric trait should not change over time.

4. Easily measured: Measurement of the biometric trait should be easy and convenient for an individual to obtain the characteristics.

Every alive person can produce the ECG signal and hence it fulfills the universal property [8]. We can also say that ECG signal is more universal as compared to any other biometric traits [2]. It is a difficult task to evaluate uniqueness property but there are certain statistical features like mean, variance and standard deviation and other features like PQRST amplitudes, DWT intervals supports that every person has dissimilar ECG. ECG signals can be recorded with minimum inconvenience and hence they are easily measured.ECG signals are also permanent in every individual. Hence, we can say that ECG signal can be used as a biometric trait.

\section{RESEARCH METHODOLOGY}

In ECG based biometric identification system there are basically four stages:

1. Data Acquisition

2. Preprocessing

3. Feature extraction

4. Classification.
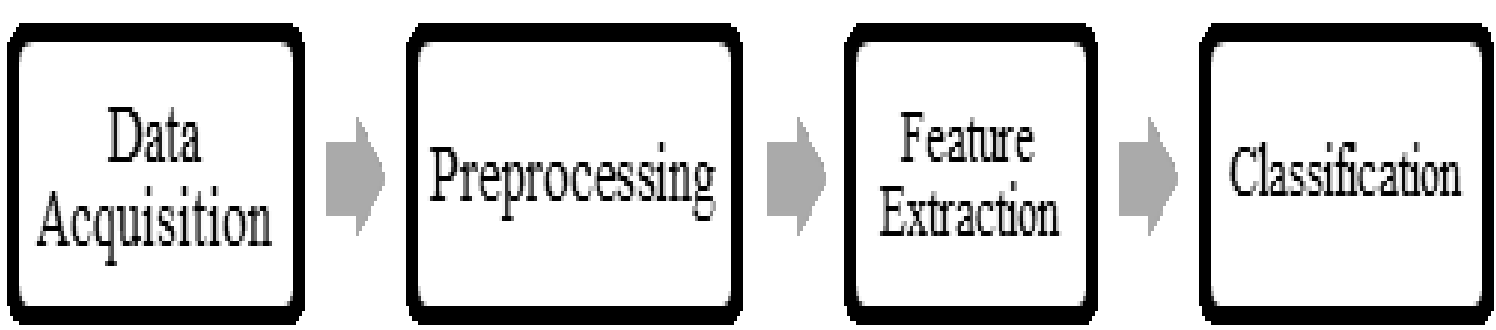

Figure 2: Basic block diagram of ECG identification system.

\section{A. Data Acquisition:}

The required ECG signal is recorded using a BIOPAC MP 100 system with Acknowledge software by placing electrodes on the human body. This data set contains ECG of 10 healthy individuals. ECG of the volunteers was recorded at rest by using a BIOPAC MP 100 system with AcKnowledge 3.9.0 software. This data set contains record of 10 subjects for 2 minutes. The volunteers were introduced about the procedure of the study and all were participated voluntarily. Multilead ECG is recorded for the analysis.

\section{B. Preprocessing:}

The next stage is preprocessing the ECG signal which contains much noise that will corrupt the signal especially when only one lead is used to detect the signal [1]. Therefore, noise reduction process has to be performed using noise filters to remove baseline wander, dc offset and high frequency interference [11][10]. The AcqKnowledge software has inbuilt filter for removing the unwanted high frequency signal. Digital FIR low pass filter with $40 \mathrm{~Hz}$ cutoff frequency is used for preprocessing step.

\section{Feature Extraction:}

In the third step, statistical features are extracted from the recorded ECG. Microsoft excel is used to calculate the feature like mean, variance and standard deviation. Other features like PQRST wave's amplitude and DWT mean intervals are calculated using Acknowledge software. These features are analyzed for each subject. Figure 3 shows the extracted features for 10 subjects. The statistical features are extracted by selecting the waveform for $10 \mathrm{sec}$ and for PQRST amplitudes, $1 \mathrm{sec}$ of lead I is selected. 


\section{UGC Approved Journal}

\begin{tabular}{|c|c|c|c|c|c|c|c|c|c|c|c|}
\hline 4 & A & $B$ & C & D & E & $\mathrm{F}$ & G & $H$ & 1 & J & K \\
\hline 1 & & P & $Q$ & $R$ & s & $T$ & Avg & Var & S.D. & DWT mean interval & \\
\hline 2 & SUBJECT 1 & $-0,09124$ & $-0,16233$ & 0.150281 & -0.82236 & 0.099019 & $-0,11121$ & 0.023128 & 0.152081 & 0.62530975 & \\
\hline 3 & SUBJECT 2 & $-0,24679$ & -0.58249 & 0.953033 & $-0,66236$ & 0,040631 & -0.27702 & 0.056268 & 0.23721 & 2.587674 & \\
\hline 4 & SUBJECT 3 & $-0,00175$ & $-0,29444$ & 1,65338 & $-1,05333$ & 0.091724 & $-0,02329$ & 0.103001 & 0.320937 & 1,680861 & \\
\hline 5 & SUBJECT 4 & 0.024195 & $-0,09557$ & 0.522351 & - $-0,61502$ & 0.10144 & $-0,01704$ & 0.015517 & 0.124567 & 0.746463 & \\
\hline 6 & SUBJECT 5 & $-0,08786$ & $-0,28005$ & 0.875126 & -0.73098 & 0.507034 & $-0,07525$ & 0.066989 & 0.258823 & 0.9879845 & \\
\hline 7 & SUBJECT 6 & 0.01196 & $-0,09037$ & 0.45309 & $-0,1625$ & $-0,00407$ & $-0,00811$ & 0.009224 & 0.096039 & 1,6626885 & \\
\hline 8 & SUBJECT 7 & $-0,03429$ & -0.19635 & 0.558309 & -0.58391 & 0.323618 & $-0,05908$ & 0.029839 & 0.172739 & 0.756247 & \\
\hline 9 & SUBJECT 8 & 0.011458 & $-0,03415$ & 0.403662 & $-0,54048$ & 0.104797 & 0,001661 & 0.011819 & 0.108714 & 0.6110275 & \\
\hline 10 & SUBJECT 9 & 0.027032 & $-0,08407$ & 0.909401 & $-0,65958$ & 0.231701 & 0,005716 & 0.030121 & 0.173555 & 2,384805 & \\
\hline 11 & SUBJECT 10 & $-0,02493$ & $-0,27292$ & 1,25272 & $-0,54513$ & 0.300317 & $-0,01735$ & 0,048162 & 0.219458 & 1,0326695 & \\
\hline & & & & & & & & & & & \\
\hline
\end{tabular}

Figure 3: Extracted Features

Figure 3 shows the excel sheet of all the extracted features for 10 subjects.

\section{Classification:}

Classification is the last step in the biometric identification system. It classifies the extracted features into the groups with similar properties. The features such as mean, variance, standard deviation are calculated to support the uniqueness property. Also when we work with large data set then these features helps to represent the complete data set with single values. DWT is taken because the ECG was very wide and we require characteristic for a fixed duration, so extraction of part of a signal is done for study of various parameters.

Here the extracted features are trained and tested using classifier. Classifier used in this research work is the artificial neural network. Ten hidden neurons are trained for 10 subjects and testing is done for 1000 epochs. The data sheet shown in figure 3 is used for the training and testing purpose.

\section{RESULTS}

ANN is used as the classifier and the data sheet shown in figure 3 is trained using neural network toolbox in MATLAB. Figure 4 shows the output in the command window. 1000 epochs are used to train and test data. The first column in the command window represents the original ECG parameter, second column is the predicted parameter and the third column shows the error in prediction which is nearly zero. Therefore the accuracy of the system is approximately $100 \%$. So the designed system also identifies the persons uniquely.

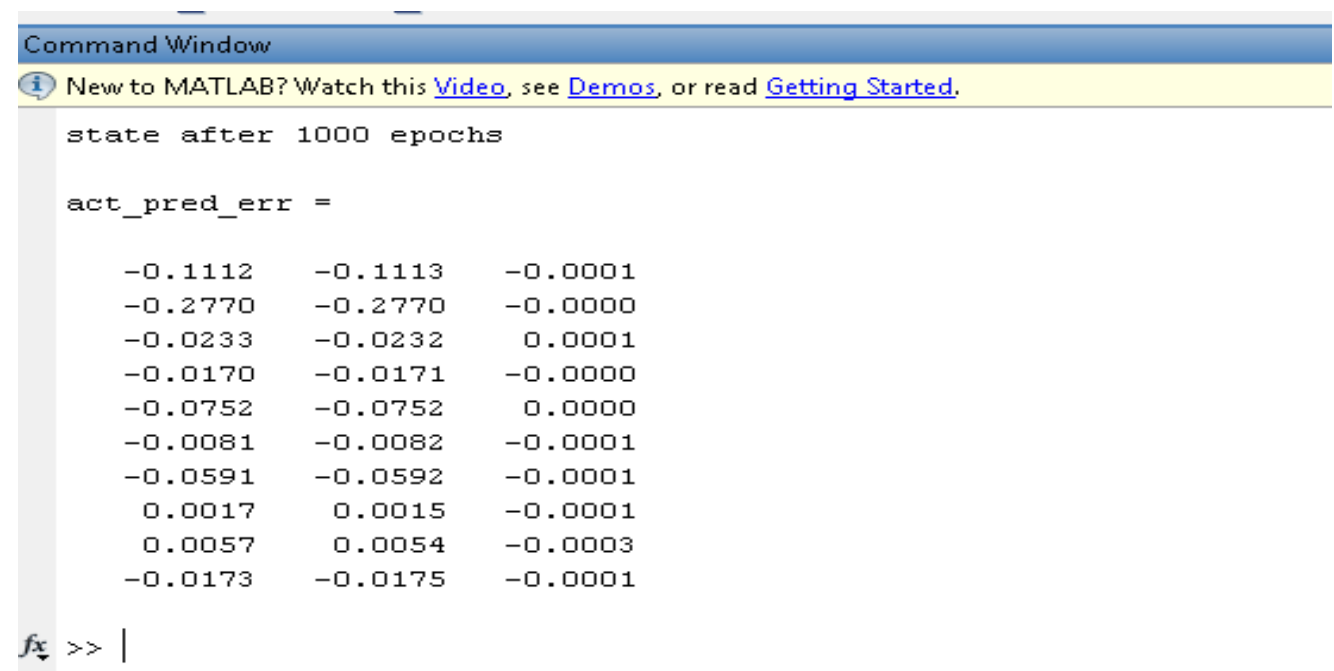

Figure 4: Command window output 


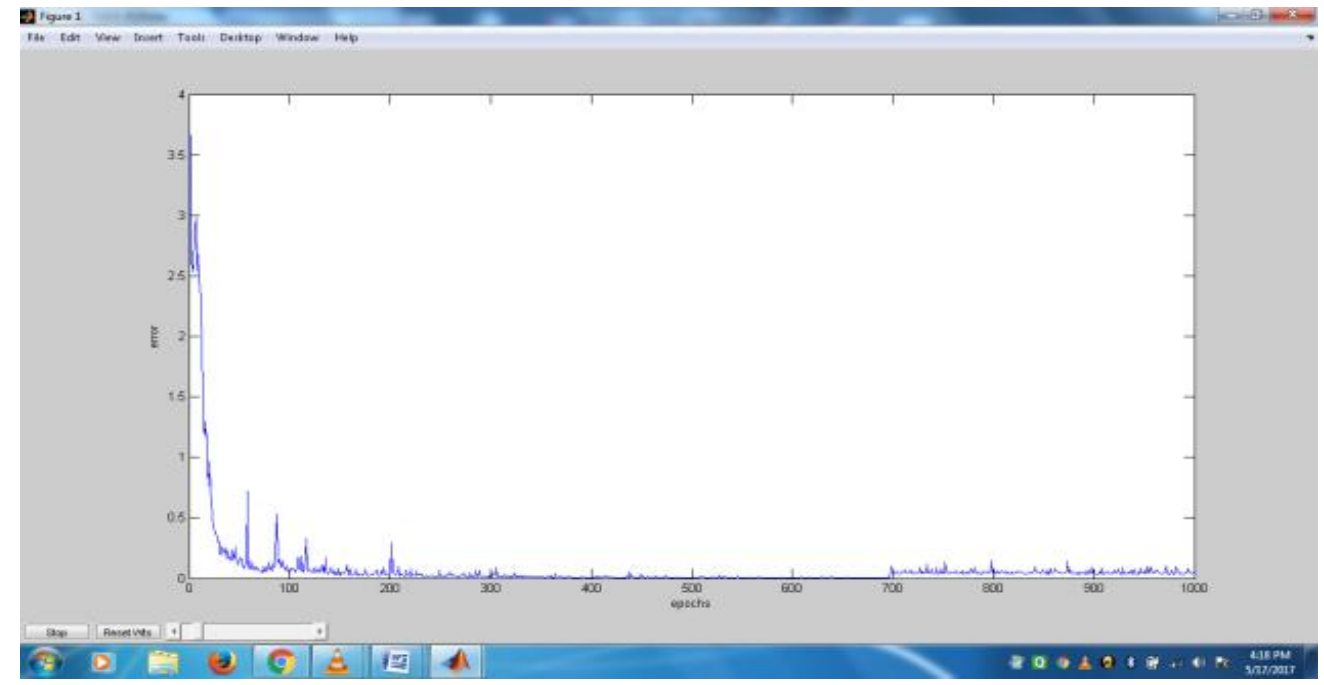

Figure 5: Error in prediction

Figure 5 shows the plot of error of prediction with the epochs. The error in prediction is calculated by subtracting the actual value from the predicted value. On the $\mathrm{x}$-axis, epochs are taken which represent the no. of iterations and on the $\mathrm{y}$-axis error is taken which represent the error in predicting the actual value. It is clearly seen that the error in prediction decreases nearly zero with the increase in epochs.

\section{CONCLUSION}

This paper deals with the analysis of ECG signal for using it in a biometric human identification system. In the beginning of the paper it was assumed that an ECG signal is used for biometric human identification as ECG possesses few biometric characteristics. From literature survey it possesses nearly all the characteristics that are essential for any biometric identification system. There is no doubt that an ECG is possessing universal, permanent and easily measured characteristics. In this research work a statistical approach is adopted to prove the uniqueness of ECG signal. Finally a classifier using Neural Network Toolbox is designed to train the neurons with the extracted features and then tested for the same. The error in the prediction of each subject is approximately zero. So the accuracy of the system is nearly $100 \%$. From the results, it is evident that an ECG signal is used for the biometric human identification system.

\section{FUTURE SCOPE}

Future Approach of this research work is to implement this system using hardware so that data acquisition process will take place in real time and after processing it will show the details of that individual. For this, large database shall be stored. Here 5 leads ECG is taken into account for Feature Extraction but in future data can be taken to a broader level by analyzing the 12 lead ECG.

\section{REFERENCES}

[1] Ainunjariah Binti Amiruddin1, Othman O. Khalifa, Performance Evaluation of Human Identification Based on ECG Signal, International Conference on Computing, Control, Networking, Electronics and Embedded Systems Engineering, 2015

[2] Babak Nasri, Mouhcine Guennoun, and Khalil El-Khatib, Using ECG as a Measure in Biometric Identification Systems, TIC-STH 2009 28-33.

[3] Carlos Carreiras, Andre Lauren, Ana Fred and Rui Ferreira, ECG Signals for Biometric Applications: Are we there yet?, 2014 11th International Conference on Informatics in Control, Automation and Robotics, 765-774.

[4] https://www.researchgate.net/figure/236342159_fig1_Figure-1-Basic-ECG-waveform-showing-PQRST-point (18/3/2017; 11:40 PM).

[5] Kirti, Manoj Duhan, A Survey Paper on the Effects of Mobile Phone Radiation on Human Heart, International Journal of Trend in Research and Development, June, 2016.

[6] Lugovaya T.S. , Biometric Human Identification based on Electrocardiogram, [Master's Thesis] faculty of computing Technologies and Informatics, Electrotechnical University "LETI",Saint-Petersburg, Russian Federation; June 2005.

[7] Newman, R., Security and access control using biometric technologies, Course Technology, 2010, 2-28.

[8] Nisha Yadav, Manoj Duhan, A Review on ECG based Biometric Human Identification, National conference on Recent Trends in Computing and Communication Technologies (RRCT-2017) ISBN: 978-93-86256-54-6, 220-224.

[9] Nisha Yadav, Manoj Duhan, ECG Signal Analysis for Biometric Characteristics, International Journal of Engineering and Technology (Communicated), May 2017.

[10] S. A. Israel, J. M. Irvine, C. Andrew, D. W. Mark and K. W. Brenda, ECG to Identify Identity Individuals, Pattern Recognition, vol. 38(1), 2005, pp. 133-142.

[11] T.W. Shen, W. J. Tompkins and Y. H. Hu, One-Lead ECG for Identity Verification, Proc. IEEE EMBS/BMES Conference, pp. 62-63, 2002. 